Orthopäde 2020 · 49:377

https://doi.org/10.1007/s00132-020-03905-9

Online publiziert: 26. März 2020

(c) Springer Medizin Verlag GmbH, ein Teil von Springer Nature 2020

Die Arthrose des Knie- und Hüftgelenks sind Volkskrankheiten mit einer zunehmenden Tendenz aufgrund der Alterung der Bevölkerung. Trotz kontinuierlicher Weiterentwicklung der Operationstechniken, des Designs und der Materialbeschaffenheit der Implantate ist dennoch die Standzeit aufgrund verschiedener Faktoren limitiert. Bei Implantatlockerung kommt nur der Austausch der gelockerten Implantatanteile infrage. Die Sineplastik oder auch die Arthrodese sind keine guten Alternativen. $\mathrm{Da}$ immer mehr Implantate eingesetzt werden, steigt auch die Anzahl der erforderlichen Revisionsoperationen. Diese Eingriffe sind neben der Belastung für den Patienten auch eine finanzielle Belastung des Gesundheitssystems. Daher ist es weiterhin das Ziel, die Implantate näher an die physiologische Situation anzupassen und die Standzeit zu verbessern. Da die knöcherne Anatomie an Knie- und Hüftgelenk anlagebedingt, entwicklungsbedingt oder auch durch operative Eingriffe oder Verletzungen durchaus sehr variabel sein kann, liegt eine Individualisierung der Implantate nahe.

Neben dem Standzeitthema hat sich ebenso gezeigt, dass trotz des grundsätzlich sehr erfolgreichen Operationsverfahrens des Gelenksersatzes eine gewisse Anzahl von Patienten mit dem erreichten Ergebnis nicht zufrieden ist und postoperativ Beschwerden behält. Dieses Phänomen ist vor allem beim Kniegelenks- und weniger beim Hüftgelenks- und Schultergelenksersatz zu verzeichnen. Je nach Literatur liegt dieser Prozentsatz bei bis zu 15-20\%. Diese Erkenntnis hat dazu geführt, dass patientenimmanente Faktoren bei der Versorgung stärker in den Vordergrund gerückt sind. Insbesondere

\author{
Christian Lüring' · Johannes Beckmann ${ }^{2}$ \\ 'Orthopädische Klinik, Klinikum Dortmund gGmbH, Dortmund, Deutschland \\ ${ }^{2}$ Department Endoprothetik untere Extremität und Fußchirurgie, Sportklinik Stuttgart, Stuttgart, \\ Deutschland
}

\title{
Individuelle Endoprothetik
}

beim Kniegelenk ist die komplexe Kinematik des Gelenks als bedeutsamer Faktor erkannt worden. Untersuchungen zeigen zweifelsfrei auf, dass vorkonfektionierte Standardimplantate bei einem hohen Prozentsatz der Patienten die natürliche Kniekinematik nicht ausreichend nachahmen. Für das Hüftgelenk gilt dies ebenso bei ca. $15 \%$ der Patienten mit Deformierungen des proximalen Femurs. Auch dieser Aspekt unterstreicht die notwendige Individualisierung von Implantaten nicht zwingend bei allen Patienten flächendeckend, aber eben bei den $\mathrm{Pa}$ tienten, die radiologisch auffällige knöcherne Verhältnisse zeigen (beim Kniegelenk z. B. sehr unterschiedliches dorsales oder distales Offset, beim Hüftgelenk z. B. das Champagnerglasfemur).

Zuletzt muss auch anerkannt werden, dass die Implantation von künstlichen Knie-, Hüft- und Schultergelenken sehr komplexe Eingriffe sind, die ein hohes Maß an operativem Geschick, Erfahrung und Genauigkeit voraussetzen. $\mathrm{Zu}$ allen Gelenken liegen hinreichend Hinweise vor, dass die Implantationsgenauigkeit aufgrund verschiedener Faktoren aber nicht bei allen Operationen umzusetzen ist, bzw. umgesetzt werden kann. Es ist auch bekannt, dass z. B. Bandinstabilitäten beim Kniegelenk, Implantatpositionsprobleme am Hüftgelenk und auch am Schultergelenk zu vermeidbaren Frührevisionen führen, die, wie schon oben erwähnt, sowohl Patient als auch das solidarische Gesundheitssystem belasten. Auch dies führt zur Erkenntnis, dass individualisierte Implantate mit entsprechenden Schablonen und Ausrichthilfen eine verbesserte Genauigkeit erzielen könnten und damit die Notwendigkeit von Frührevisionen vermeiden helfen.
Das vorliegende Themenheft hat die Aufgabe, für alle genannten Gelenke die individuellen Gründe für eine Individualisierung aufzuarbeiten und den aktuellen Sach- und Erkenntnisstand der Literatur aufzuarbeiten.

Viel Spaß beim Lesen wünschen

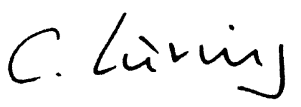

Prof. Dr. Christian Lüring und

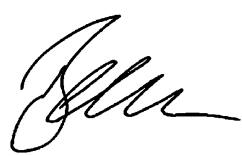

Prof. Dr. Johannes Beckmann

\section{Korrespondenzadresse}

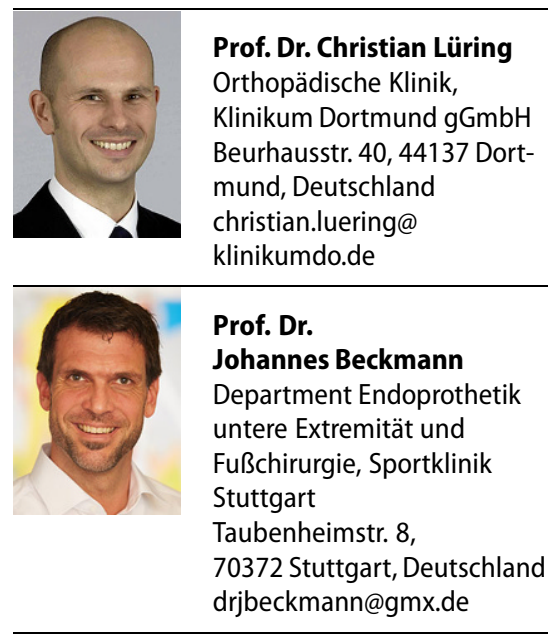

Interessenkonflikt. J. Beckmann: Conformis. C. Lüring gibt an, dass kein Interessenkonflikt besteht. 